\title{
Plasmacytoid DCs From Patients With Sjögren's Syndrome Are Transcriptionally Primed for Enhanced Pro-inflammatory Cytokine Production
}

OPEN ACCESS

Edited by: Keith Elkon, University of Washington, United States

Reviewed by: Tomas Mikael Mustelin, University of Washington,

United States

lan P. Giles,

University College London,

United Kingdom

*Correspondence:

Timothy R. D. J. Radstake

t.r.d.j.radstake@umcutrecht.nl

†These authors share first authorship

¥These authors share last authorship

Specialty section:

This article was submitted to Autoimmune and Autoinflammatory

Disorders,

a section of the journal

Frontiers in Immunology

Received: 24 April 2019

Accepted: 20 August 2019 Published: 04 September 2019

Citation:

Hillen MR, Pandit A, Blokland SLM, Hartgring SAY, Bekker CPJ, van der Heijden EHM, Servaas NH,

Rossato M, Kruize AA, van Roon JAG and Radstake TRDJ (2019)

Plasmacytoid DCs From Patients With

Sjögren's Syndrome Are

Transcriptionally Primed for Enhanced

Pro-inflammatory Cytokine

Production. Front. Immunol. 10:2096.

doi: 10.3389/fimmu.2019.02096
Maarten R. Hillen ${ }^{1,2 \dagger}$, Aridaman Pandit ${ }^{1,2+}$, Sofie L. M. Blokland ${ }^{1,2}$, Sarita A. Y. Hartgring ${ }^{1,2}$, Cornelis P. J. Bekker ${ }^{1,2}$, Eefje H. M. van der Heijden ${ }^{1,2}$, Nila H. Servaas ${ }^{1,2}$, Marzia Rossato ${ }^{1,2,3}$, Aike A. Kruize ${ }^{2}$, Joel A. G. van Roon ${ }^{1,2 \ddagger}$ and

Timothy R. D. J. Radstake ${ }^{1,2 * \neq}$

${ }^{1}$ Laboratory of Translational Immunology, University Medical Center Utrecht, Utrecht University, Utrecht, Netherlands, ${ }^{2}$ Department of Rheumatology and Clinical Immunology, University Medical Center Utrecht, Utrecht University, Utrecht, Netherlands, ${ }^{3}$ Department of Biotechnology, University of Verona, Verona, Italy

Primary Sjögren's syndrome (pSS) is a systemic auto-immune disease typified by dryness of the mouth and eyes. A majority of patients with pSS have a type-I interferon (IFN)-signature, which is defined as the increased expression of IFN-induced genes in circulating immune cells and is associated with increased disease activity. As plasmacytoid dendritic cells (pDC) are the premier type-I IFN-producing cells and are present at the site of inflammation, they are thought to play a significant role in pSS pathogenesis. Considering the lack of data on pDC regulation and function in pSS patients, we here provided the first in-depth molecular characterization of pSS pDCs. In addition, a group of patients with non-Sjögren's sicca (nSS) was included; these poorly studied patients suffer from complaints similar to pSS patients, but are not diagnosed with Sjögren's syndrome. We isolated circulating pDCs from two independent cohorts of patients and controls (each $n=31$ ) and performed RNA-sequencing, after which data-driven networks and modular analysis were used to identify robustly reproducible transcriptional "signatures" of differential and co-expressed genes. Four signatures were identified, including an IFN-induced gene signature and a ribosomal protein gene-signature, that indicated pDC activation. Comparison with a dataset of in vitro activated pDCs showed that pSS pDCs have higher expression of many genes also upregulated upon pDC activation. Corroborating this transcriptional profile, pSS pDCs produced higher levels of pro-inflammatory cytokines, including type-I IFN, upon in vitro stimulation with endosomal Toll-like receptor ligands. In this setting, cytokine production was associated with expression of hub-genes from the IFN-induced and ribosomal protein gene-signatures, indicating that the transcriptional profile of pSS pDCs underlies their enhanced cytokine production. In all transcriptional analyses, nSS patients formed an intermediate group in which some patients were molecularly similar to pSS patients. Furthermore, we used the identified transcriptional signatures to develop a 
discriminative classifier for molecular stratification of patients with sicca. Altogether, our data provide in-depth characterization of the aberrant regulation of pDCs from patients with nSS and pSS and substantiate their perceived role in the immunopathology of pSS, supporting studies that target pDCs, type-I IFNs, or IFN-signaling in pSS.

Keywords: Sjögren's syndrome, plasmacytoid dendritic cells, type-I interferon, transcriptomics, gene network analysis

\section{INTRODUCTION}

Primary Sjögren's syndrome (pSS) is a systemic rheumatic autoimmune disease characterized by mononuclear infiltration of the exocrine glands, associated with dysfunction and destruction of the glands and dryness of primarily mouth and eyes (1). Patients experience debilitating fatigue, can suffer from systemic manifestations that can involve a wide range of organs, and have an increased chance of lymphoma (2). At this time, no effective treatment is available and a better understanding of immunopathology is needed to provide novel therapeutic targets.

An interferon (IFN)-signature, defined as the increased expression of a panel of type-I IFN induced genes (IFIG; e.g., MX1, IFIT3, IFI44), is present in the majority of pSS patients and is associated with increased systemic disease activity $(3,4)$. Type-I IFNs stimulate monocytes to produce increased levels of $B$ cell activating factor (BAFF), an important mediator in pSS that drives B cell activation and auto-antibody production (4). In addition, deficiency for the type-I IFN receptor in mice prevents experiment Sjögren's syndrome (5). As such, type-I IFNs are considered to be crucial mediators in pSS pathogenesis $(6,7)$.

Plasmacytoid dendritic cells (pDCs) are uniquely capable of rapidly producing high levels of type-I IFN upon activation. pDCs constitutively express endosomal Toll-like receptors (TLR) 7 and 9, through which they recognize viral RNA and DNA but can also bind endogenous nucleic acids. Triggering of these TLRs results in production of type-I IFNs or other pro-inflammatory cytokines, depending on the cellular compartment in which the receptors encounter their ligand (8). Importantly, type-I IFNs also act on pDCs themselves, and autocrine or paracrine IFN primes them for enhanced IFN-production and potently amplifies their responses (9-11). As self-nucleic acids, in the form of autoantibody-complexes and apoptotic cell material, are present in patients with pSS and can strongly activate pDCs via TLR7 and TLR9, these cells are thought to be a major source of type-I IFN in pSS (12-14).

In patients with pSS, numbers of pDCs in peripheral blood are decreased and associated with increased salivary gland inflammation $(15,16)$. Furthermore, pDCs infiltrate the pSS salivary glands and local pDC numbers correlate with the number of IFN- $\alpha$ producing cells $(15,17,18)$. Moreover, pDCs from IFN signature-positive pSS patients have increased expression of TLR7 and RNA-sensing receptors, identifying pathways via which these cells can be activated to produce enhanced type-I IFNs (19). Thus, a considerable body of evidence supports an important role for $\mathrm{pDCs}$ in pSS pathogenesis. Despite this, there is a lack of data on purified pDCs from pSS patients, at least in part due to their scarcity ( $<0.5 \%$ of leukocytes). In addition,
pDCs could possibly play a role in (a subset of) patients with non-Sjögren's sicca (nSS): this poorly studied group suffers from unexplained dryness similar to patients with pSS, but is not diagnosed with Sjögren's syndrome by the clinician and does not meet the classification-criteria. Comparing the transcriptional profile of pSS and nSS pDCs with those from healthy donors can provide a better understanding of their role in pathogenesis and aid in molecular patient stratification (20).

\section{MATERIALS AND METHODS}

\section{Patients and Controls}

All pSS patients met the 2002 AECG classification-criteria (21). nSS patients presented with dryness-complaints without a known cause, did not have any generalized autoimmune disease including pSS as evaluated by an experienced rheumatologist, and did not fulfill the classification-criteria for pSS. Fourteen nSS patients were clinically re-evaluated several years after initial sample collection; labial biopsy, assessment of laboratory parameters, and physical examination were all performed at follow-up and the 2016 ACR-EULAR criteria (22) were used for classification. For in vitro experiments, independent donors were included (Table 1). The medical ethics committee of the UMC Utrecht approved the study (METC nr 13-697), all patients gave their written informed consent.

\section{Cell Isolation and RNA Isolation}

Peripheral blood mononuclear-cells were isolated from heparinized peripheral blood by density centrifugation using Ficoll-Paque Plus (GE Healthcare). pDCs were freshly isolated by MACS using BDCA-4+ isolation kit (Miltenyi Biotec). In addition, monocytes were freshly isolated by MACS from a subset of 43 donors (14 HC, 10 nSS, 19 pSS; randomly selected from either cohort) using the CD14+ isolation kit (Miltenyi) after isolation of pDCs to quantify the IFN-score. Cells were lysed in RLTplus buffer (Qiagen Allprep Universal kit) supplemented with beta-mercaptoethanol (final concentration $1 \%$ ) for transcriptional analyses. Total RNA was purified using AllPrep Universal Kit (Qiagen), according to the manufacturer's instructions. RNA concentration was assessed with Qubit RNA Kit (Thermo Fisher Scientific) and RNA integrity was measured by capillary electrophoresis using a Bioanalyzer (Agilent) and the RNA 6000 Nano Kit (Agilent Technologies); all samples had RIN-score $>7.0$.

\section{RNA Sequencing and Analysis}

For the discovery cohort samples, RNA sequencing was performed at the Beijing genomics institute on a NextSeq 


\begin{tabular}{|c|c|c|c|c|c|c|c|c|}
\hline & \multicolumn{6}{|c|}{ RNA sequencing } & \multicolumn{2}{|c|}{ In vitro experiments } \\
\hline & \multicolumn{3}{|c|}{ Discovery cohort $(n=31)$} & \multicolumn{3}{|c|}{ Replication cohort $(n=31)$} & \multirow[b]{2}{*}{$\mathrm{HC}$} & \multirow[b]{2}{*}{ pSS } \\
\hline & $\mathrm{HC}$ & nSS & pSS & $\mathrm{HC}$ & nSS & pSS & & \\
\hline $\mathrm{N}[\mathrm{M} / \mathrm{F}]$ & $8[0 / 8]$ & $9[0 / 8]$ & $14[3 / 11]$ & $9[0 / 9]$ & $11[0 / 11]$ & $11[0 / 11]$ & $17[0 / 17]$ & $22[2 / 20]$ \\
\hline Age (year) & 58 [54-59] & 43 [34-61] & 54 [44-61] & 51 [48-55] & 45 [37-60] & 55 [40-63] & 54 [47-57] & 54 [47-68] \\
\hline LFS (foci/4 mm²) & - & $0.0[0.0-0.0]$ & $1.9[1.1-3.0]$ & - & $0.1[0.0-0.5]$ & $2.2[1.5-3.0]$ & - & $2.0[1.3-3.0]$ \\
\hline ESSDAI & - & - & $2[1-6]$ & - & - & $5[3-11]$ & - & $5[2-10]$ \\
\hline ESSPRI & - & - & $4[2-7]$ & - & - & $5[3-7]$ & - & $6[4-7]$ \\
\hline Schirmer (mm/5 min) & - & $3[1-14]$ & $5[2-15]$ & - & 8 [3-18] & $12[6-23]$ & - & $3[0-8]$ \\
\hline ANA (no. positive [\%]) & - & $1[11 \%]$ & $10[71 \%]$ & - & 5 [45\%] & 9 [82\%] & - & 18 [82\%] \\
\hline SSA (no. positive [\%]) & - & 2 [22\%] & $8[57 \%]$ & - & 3 [27\%] & 7 [64\%] & - & $17[77 \%]$ \\
\hline SSB (no. positive[\%]) & - & $0[\%]$ & 4 [29\%] & - & $0[0 \%]$ & 3 [27\%] & - & 12 [55\%] \\
\hline Serum lgG (g/L) & - & $12[8-14]$ & $14[10-18]$ & - & $13[10-14]$ & 18 [10-33] & - & $14[8-17]$ \\
\hline ESR (mm/hour) & - & $11[7-15]$ & $11[6-25]$ & - & 8 [6-15] & 14 [8-34] & - & 17 [7-34] \\
\hline $\mathrm{CRP}(\mathrm{mg} / \mathrm{L})$ & - & $1[0-4]$ & $1[1-7]$ & - & $1[0-2]$ & $2[1-3]$ & - & $2[1-6]$ \\
\hline Treatment (no. positive [\%]) & - & $1[11 \%]$ & 3 [21\%] & - & 1 [10\%] & $4[36 \%]$ & - & 5 [23\%] \\
\hline
\end{tabular}

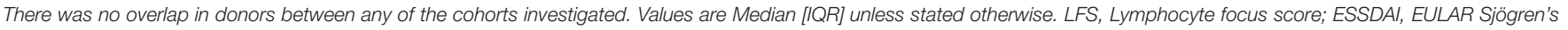

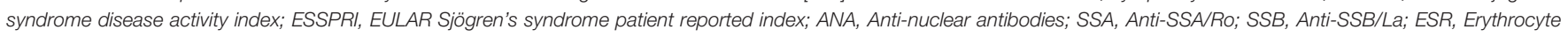
sedimentation rate; CRP, C-reactive protein. Immunosuppressive treatment included (combinations of) hydroxychloroquine $(n=10)$, prednisone $(n=4)$, azathioprine $(n=5)$.

500 sequencer (Illumina) by applying standard manufacturer's protocols. About 20 million paired-end (91 bp) reads were generated for each sample. For the replication cohort, RNA sequencing was performed at the Beijing genomics institute using an Illumina HiSeq 4000 sequencer (Illumina), by applying standard manufacturer's protocols. About 20 million pairedend (100 bp) reads were generated for each sample. Raw reads obtained from RNAseq were quality checked using the FastQC tool (https://www.bioinformatics.babraham.ac.uk/ projects/fastqc/). All samples passed the quality check. For each sample, reads were then aligned to the human genome assembly (GRCh38 build 79) (23) using STAR aligner (24). The aligned reads (mapping quality $>30$ ) were used to calculate the read counts using Python package HTSeq (25) for each annotated gene. Since samples were collected, isolated and stored at different timepoints and by different individuals, this can create batch effects in the RNASeq data. We used RUVSeq (26) to remove unwanted variance ( $k=1$ parameter) for both cohorts and used RUVSeq-corrected read counts for further analysis. To approximate the biological variability and overdispersion found in the RNASeq data, the batch-corrected read counts were modeled as a negative binomial distribution to identify differentially expressed genes (DEGs) using Bioconductor/R package DESeq2 (27). We used Wald's test to identify DEGs in each pair-wise comparison performed between the three groups (HC, nSS, and pSS) and used likelihood ratio test (LRT) to identify DEGs considering multiple groups. Differences in gene expression with a nominal $p$-value of $<0.05$ were considered differentially expressed. Variance stabilizing transformation was applied to the raw read count data to obtain normalized gene counts (variance stabilized data or VSD), which were used for subsequent analyses.
To investigate if the pSS pDCs were similar to TLR-activated cells, we compared their gene expression profiles with the expression signature of in vitro stimulated $\mathrm{pDCs}$ using gene set enrichment analysis (GSEA) (28). For this, we used a publically available RNA-sequencing dataset of TLR-ligand stimulated isolated pDCs (29). Bioconductor/R package FGSEA was used to perform the GSEA analysis and compare the genes differentiallyexpressed in the pSS pDCs to those differential upon stimulation with pRNA or CPG-P, which was done separately for the up and down-regulated genes (30). Ten thousand permutations were performed to calculate the significance of enrichment scores and FDR-corrected $p$-values.

\section{Weighted Gene Co-expression Network Analysis (WGCNA)}

To study the inter-dependence between genes and their interactions, we constructed two gene co-expression networks (one for the discovery cohort and another for the replication cohort) using WGCNA Bioconductor/R package (31). The gene co-expression networks were constructed using all the genes that were differentially expressed $(\mathrm{p}<0.05)$ either in the pairwise analysis (Wald's test) or in the multi-group comparison (LRT). The modules were defined by first performing an unsigned pairwise spearman's correlation between genes and subsequently transforming the co-expression similarities into an adjacency matrix (that provides the relative connection strengths between genes) and scaling the adjacency matrix to achieve a scale-free topology (scaling power $=6$, selected to have a network that fits the scale-free topology criterion).

For each module the gene or the first principal component was calculated and plotted using ggplot 2 and ggsci libraries in R. For each gene, the connectivity represents the sum of connection 
strengths (or the weighted correlations) of each node with its neighbors in a given module. We normalized the connectivities for each module in the range $[0,1]$, where 1 represents the maximum and 0 represents the minimum connectivity in the module. We calculated the overlap between the modules constructed from the two independent cohorts and used Fisher's exact test to identify modules with significant overlaps (32). We performed pathway enrichment analysis on replicated DEGs found in modules exhibiting significant overlap in the two cohorts using R/Bioconductor package ReactomePA (33). The networks were plotted using Cytoscape (version 3.6.1) (34).

\section{pDC Culture and Cytokine Analysis}

Isolated pDCs were cultured in RPMI Glutamax (Gibco) supplemented with penicillin, streptomycin (both Gibco), and $10 \%$ FBS (Sigma). Cells were cultured at $6^{*} 10^{5}$ cells $/ \mathrm{mL}$ in 96 wells round-bottomed plates (Thermo Fisher Scientific) in the presence of $1 \mathrm{mM}$ of loxoribine (TLR7L; InvivoGen) or $1 \mu \mathrm{M}$ of CPG-C (TLR9L; InvivoGen). After $3 \mathrm{~h}$, supernatants were harvested and cells lysed, samples were stored at $-80^{\circ} \mathrm{C}$. Cytokines were measured in the supernatants using multiplex immunoassay.

\section{Quantitative PCR}

Quantitative-PCRs were performed in duplicate per sample using SYBR Select Master Mix (Applied Biosystems) and the Quantstudio system (Thermo Fisher Scientific). In the cultured pDCs, mRNA expression was measured and normalized to the mean expression of two housekeeping genes: GAPDH and GUSB (Supplementary Table 1). The relative fold change (FC) of each sample was calculated in relation to the $\Delta \mathrm{Ct}$ of a random unstimulated sample in the $\mathrm{HC}$ group (reference) according to the formula $\mathrm{FC}=2^{-\Delta \Delta \mathrm{Ct}}$, where $\Delta \Delta \mathrm{Ct}=\Delta \mathrm{Ct}$ sample$\Delta \mathrm{Ct}$ reference.

To determine the IFN-score, the relative expression of five IFN-induced genes (IFI44L, IFI44, IFIT3, LY6E, and MX1) was assessed using qPCR. Expression of each sample was normalized to the mean expression of two housekeeping genes: GAPDH and GUSB (Supplementary Table 1). The IFN-score was calculated as previously described (4) and divided by the number of genes measured (five).

\section{Flow Cytometry}

To confirm consistent purity of isolated cells, the isolated cell fraction was stained and analyzed using a FACS Canto II or LSRFortessa flow cytometer (both BD). The purity of pDCs was (median [IQR]) 92\% [87-95\%], monocyte purity was $97 \%$ [95-98\%]; there were no differences in purity between any of the groups. For assessment of CCR5-expression on pDCs, 1 million PBMCs were stained for $30 \mathrm{~min}$ at $4^{\circ} \mathrm{C}$ and the expression of CCR5 on lineage-negative (CD3-CD14-CD16-CD19-CD56-) and HLA-DR+CD123+BDCA-2+ pDCs was determined (Supplementary Table 2).

\section{Prediction Model}

We used the RUVSeq Bioconductor/R package (26) to consider deviance in the residuals $(k=1)$ from a GLM regression of the counts to batch-corrected read counts from discovery and replication cohorts. We used the caret Bioconductor/R package to perform backward feature selection and 10-fold cross validation to get high prediction accuracy. This allowed us to select 467 genes. We further used the SVM algorithm (svmRadial method from caret $\mathrm{R}$ package) to develop a classifier using the 467 selected genes to discriminate pSS patients from HC donors.

\section{Statistical Analysis}

For RNA-sequencing analysis, we used Wald's test to identify DEGs in each pair-wise comparison performed between the three groups (HC, nSS, and pSS) and used LRT to identify DEGs considering multiple groups. For the in vitro experiments, differences between patients and controls were assessed using Mann-Whitney $U$-test. Changes in expression of ribosomal proteins after stimulation were analyzed using Wilcoxon matched-pairs signed rank test. Correlations were assessed using Spearman's rank correlation coefficient. Two-sided testing was used for all analyses. Differences and correlations were considered to be significant at $p<0.05$. As the gene signatures identified were replicated in an independent cohort, no FDRcorrection was used for the RNA-sequencing analysis.

\section{RESULTS}

\section{pSS and nSS pDCs Are Transcriptionally Distinct From HC pDCs and Analogous to TLR-Stimulated Cells}

We recruited two independent cohorts of patients and healthy controls (discovery and replication, each $n=31$; Table 1) and compared the transcriptome of their circulating pDCs. The discovery cohort revealed a large number of differentially expressed genes (DEGs) between pSS pDCs and $\mathrm{HC}$ pDCs (Figure 1A). Similarly, in the replication cohort we observed a large number of DEGs between pSS and HC (Figure 1D). nSS pDCs exhibited alterations concordant with pSS pDCs in both cohorts, and a substantial fraction of DEGs in pSS pDCs were also differentially expressed in nSS pDCs (47\% and $23 \%$ in discovery and replication cohort, respectively) (Figures 1A,D). In addition, genes differentially expressed in both pSS and nSS pDCs mostly exhibited the same directionality (>99\% of DEG in discovery and replication). However, the magnitude of differences in expression was generally larger in pSS pDCs compared to nSS pDCs (Figures 1B,E). As such, we also observed a substantial number of DEGs between pSS and nSS patients (Figures 1A,D). Multivariate analysis using DEGs from either cohort corroborated this observation; the transcriptional profiles of pDCs from pSS and nSS patients were distinct from HCs, but not necessarily from each other (Figures 1C,F).

Given the observed large transcriptional changes and the fact that pSS is a systemic disease, we hypothesized that the transcriptome of pSS pDCs indicated systemic activation. To test this, we compared the alterations identified in pSS pDCs with publically available transcriptional data from $\mathrm{pDCs}$ activated with ligands for TLR7 and TLR9 (29). Gene set enrichment analysis confirmed that a relatively large number of 

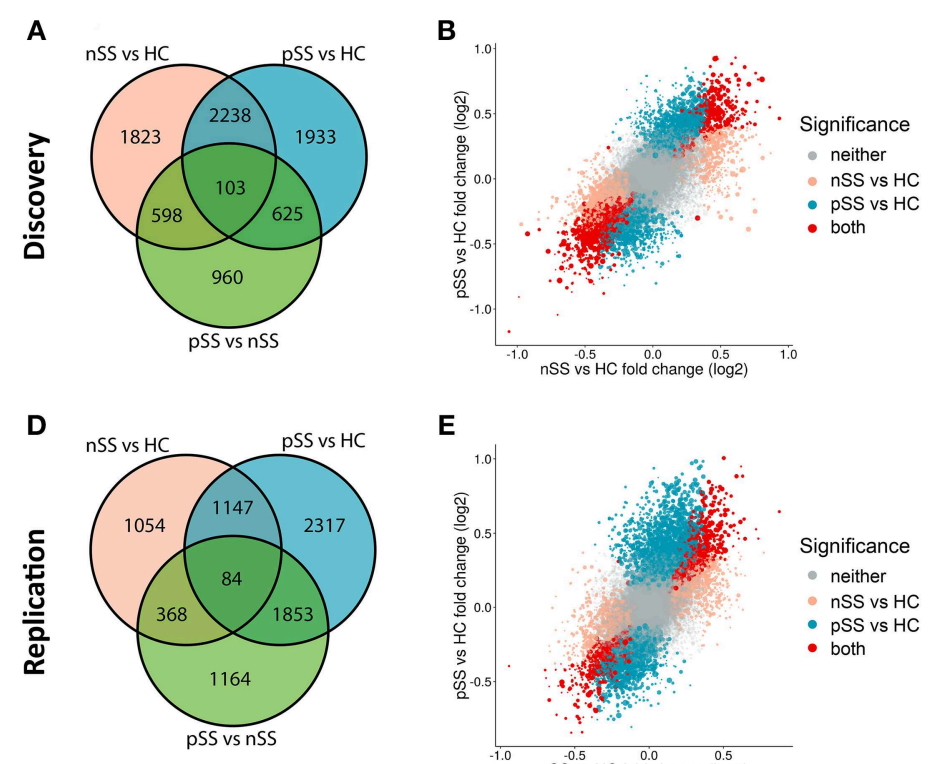

E

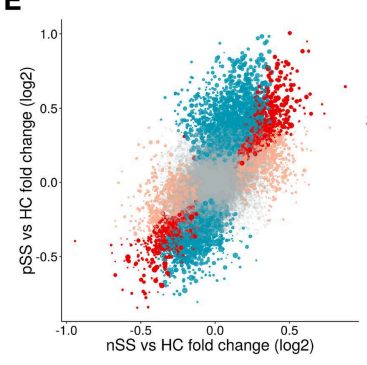

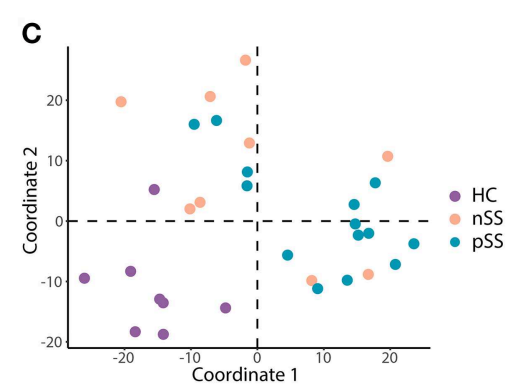

$\mathbf{F}$

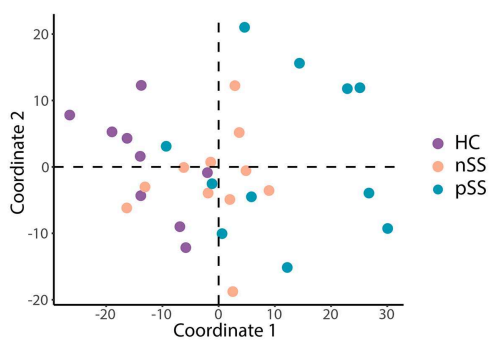

Replication
G

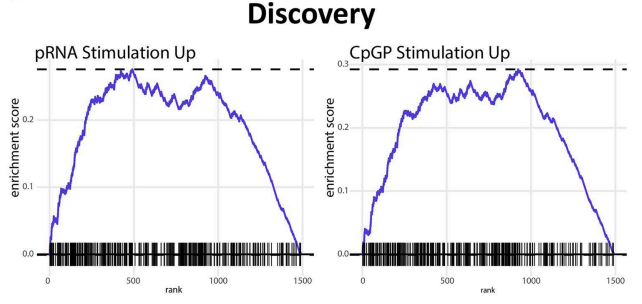

H
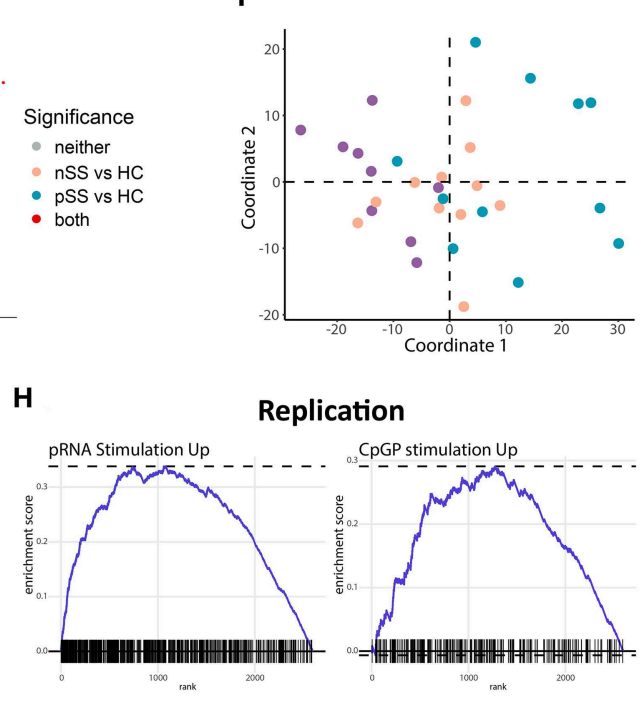

FIGURE 1 | Circulating pDCs from pSS and nSS patients are transcriptionally altered and display gene-expression patterns similar to TLR-stimulated pDCs. pDCs were isolated from peripheral blood of two independent cohorts; discovery and replication (each $n=31$ ). RNA sequencing was performed independently for both cohorts. Genes differentially expressed (nominal $p$-value $p<0.05$ ) between any of the three groups from the discovery cohort were identified (A). The association between gene expression in pSS vs. $\mathrm{HC}$ and nSS vs. $\mathrm{HC}$ was plotted; each gene is represented as a dot, with the color reflecting the comparison in which it is significantly differentially expressed and the size reflecting the expression level (larger dots depict genes with higher expression) (B). Multi-dimensional scaling was used to plot the dissimilarities between the donors from the discovery cohort based on the expression of all differentially expressed genes, taking into account all comparisons (C). The same analyses were performed on RNA sequencing data from the replication cohort (D-F). Using gene-set enrichment analysis, the differentially-expressed upregulated genes in pSS pDCs as compared to $\mathrm{HC}$ were compared to those of in vitro stimulated pDCs from a published study (29). There was a significant overlap in upregulated genes between pDCs stimulated with either pRNA (TLR7 ligand) and CPG-P (TLR9 ligand) and pSS pDCs (all FDR-corrected $p<0.05 ; \mathbf{G}, \mathbf{H})$.

DEGs found in pSS pDCs was also differentially expressed in the stimulated pDCs (Figures 1G,H, Supplementary Figure 1). Thus, the alterations in circulating pDCs from pSS patients are consistent with those found in activated cells.

To identify the genes most robustly and consistently altered, those differentially expressed in both cohorts were selected (Figure 2A). As material from the two cohorts was independently collected, sequenced, and analyzed, we considered these genes to be replicated. Examples of replicated genes are depicted in Figure 2B. These include upregulation of TRIM21/Ro52, which is the target of Sjögren's-specific autoantibodies; the decreased expression of a large set of ribosomal proteins (RPs) including $R P L 23$; and the increased expression of C-C chemokine receptor 5 (CCR5), which is an important driver of pDC migration under inflammatory conditions (35). Validation of this latter observation by assessment of the surface-expression of CCR5 using flow-cytometry confirmed the increased expression of
CCR5 on pSS pDCs compared to HCs (Figures 2C,D). In addition, a large number of IFN-induced genes including IFIT3 was upregulated in pSS and, to a lesser extent, nSS patients, including most genes previously reported to be differentially expressed in pDCs from IFN-signature positive pSS patients (19) (Figure 2B, Supplementary Figure 2). Furthermore, we observed consistent upregulation of TLR2, TLR4, and TLR8 in pSS (Supplementary Figure 2). In general, changes in single genes were most clear in pSS while the nSS patients displayed an intermediate phenotype.

\section{Network Analysis Identifies Functional Signatures of Differentially Expressed Genes}

Genes that share a molecular function have been shown to exhibit correlated changes in their expression profiles (32). To identify 

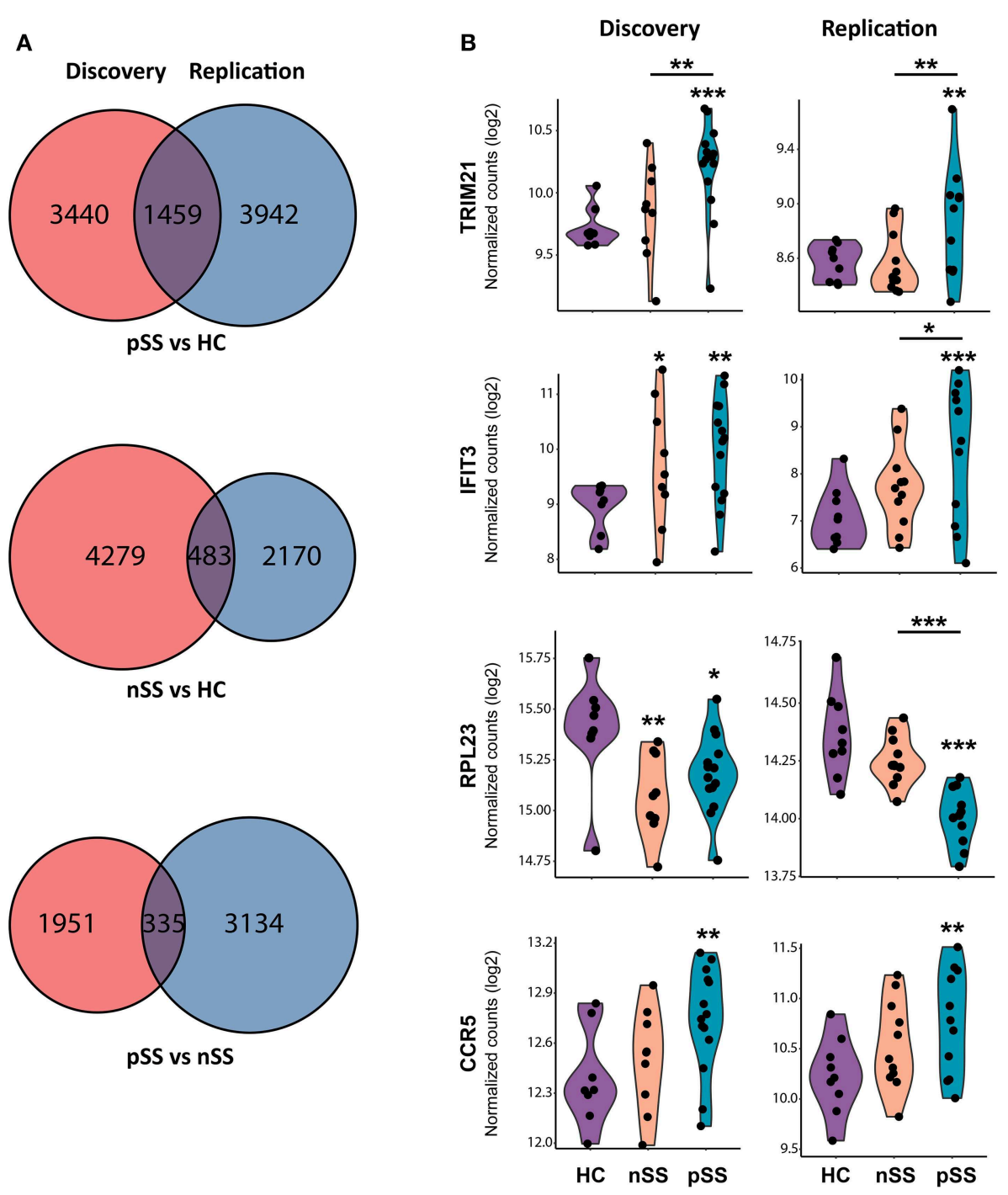

C

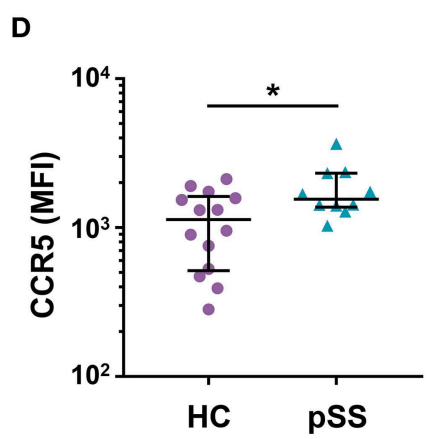

FIGURE 2 | A large set of genes is consistently differentially expressed in patients and CCR5 is increased on the surface of pSS pDCs. Venn diagrams showing the genes differentially expressed (nominal $p$-value $<0.05$ ) in the discovery and replication cohort for the three group comparisons (A). Violin plots of a selection of differentially expressed genes are depicted; expression is shown in normalized read counts, which is plotted on a 2 log axis. (B). Mononuclear cells were isolated from peripheral blood of $\mathrm{HC}$ and pSS donors and the expression of CCR5 on the surface of lineage-negative and HLA-DR ${ }^{+} \mathrm{CD} 123^{+} \mathrm{BDCA}-2^{+}$pDCs from pSS patients $(n$ $=10)$ and $\mathrm{HC}(n=14)$ was assessed using flow-cytometry. A representative histogram from one $\mathrm{HC}$ and pSS donor is depicted in (C), fluorescence minus one (FMO) controls are indicated with a dotted line. Median fluorescence intensity (MFI) is plotted (D). *, ${ }^{\star \star}$, and ${ }^{\star \star \star}$ represent $p<0.05, p<0.01$, and $p<0.001$, respectively. 
the key pathways altered in the patient pDCs, we constructed gene-correlation networks using WGCNA. This method allows the identification of modules of strongly co-expressed genes and their transcriptional hubs. We identified 8 modules from the discovery cohort (DMs) and 12 modules from the replication cohort (RMs) (Supplementary Figure 3). To find the most robust and reproducible modules, we assessed the extent of overlap between the modules from the two cohorts. Five modules from the discovery cohort exhibited significant overlap with four modules from the replication cohort (Figure 3A). The genes in these replicated modules were designated as "gene signatures" gray (DM1:RM6), blue (DM2:RM9), yellow (DM4:RM11), and red (DM6+DM7:RM1). These replicated signatures represent sets of genes that are not only differentially expressed, but also coexpressed in two independent cohorts of patients and controls, emphasizing their consistency and importance.

Functional annotation indicated that the gene signatures were associated with translation and nonsense-mediated decay (blue), transcription initiation and regulation (yellow), and IFN-signaling (red) (Figure 3B). No pathways were enriched for the gray gene signature. Genes from the red and yellow signatures were increased in nSS and pSS patients, the blue signature genes were decreased in patients compared to $\mathrm{HC}$. Again, the nSS patients typically showed gene expression intermediate between the HC and pSS groups (Figures 3C-E, Supplementary Figure 4).

\section{Identification of Hub-Genes in Identified Signatures}

To further close in on the most relevant differentially-expressed genes and processes within the signatures, we identified their hub-genes using the normalized connectivity of each gene in both cohorts (Figure 4A). Connectivity indicates the number and strength of correlations each gene has with the other genes in the module. Genes with high connectivity ( $>75$ th percentile) in both cohorts were designated as hub and their connections were plotted (Figure 4B, Supplementary Figure 4). Hub-genes in the red signature were involved in signaling pathways downstream of the IFN- $\alpha / \beta$ receptor and their upregulation was associated with antiviral processes and pDC activation. Genes encoding ribosomal proteins were hub-genes in the blue signature; transcription-regulation genes were hub-genes in the yellow signature. Genes associated with type-I IFN-activity and pDC activation exhibited the largest fold-changes in pSS patients compared to HCs (Figure 4B).

As the red signature hub-genes contained four of the five genes used to quantify the IFN-score (4), we expected this signature to be associated with the IFN-score. To confirm this, we quantified the IFN-score using qPCR on circulating monocytes from 43 of the donors studied (randomly selected). We found a very strong correlation between the monocyte IFN-score and IFIG expression in pDCs, both on the level of individual genes and the composite IFN-score (Supplementary Figure 5A). Thus, the red signature genes indeed strongly correlate with the IFN-score and suggest signaling downstream from the type-I IFN receptor in patient $\mathrm{pDCs}$, associated with $\mathrm{pDC}$ activation (9-11). In addition to described IFIGs (19) the red gene signature reveals a large set of novel genes associated with the IFN-signature in pSS pDCs.

\section{Low Ribosomal Protein Gene Expression Reflects in vivo pDC Activation}

The blue signature represents broadly decreased expression of ribosomal proteins. Classical DCs decrease their transcription and translation of RPs upon stimulation with various activating signals, including TLR ligands (36-38). To investigate the potential link between the decreased expression of RPs in the blue signature and pDC activation, we went back to the RNA sequencing data from the TLR-triggered pDCs (29) and assessed the expression of all expressed RP genes. Stimulation with IL-3 downregulated nearly all RPs, the addition of FSME (primarily a TLR7 ligand) further downregulated the expression of these genes (Figure 5A).

To confirm that activation of pDCs causes broad downregulation of RPs, we cultured pDCs from pSS and $\mathrm{HC}$ donors for $3 \mathrm{~h}$ in the presence of ligands for TLR7 (loxoribine) or TLR9 (CPG-C) and measured the expression of three hub-genes from the blue gene signature ( $R P L 11, R P L 27, R P S 11)$. Indeed, all three RPs were downregulated upon stimulation with loxoribine in $\mathrm{HC}$ pDCs, while RP expression in pSS patients was typically lower compared to the HC cells consistent with the sequencing data (Figure 5B). Thus, the decreased expression of RPs in the blue signature seems to reflect in vivo $\mathrm{pDC}$ activation, possibly via TLR7 triggering.

\section{pSS pDCs Produce Increased Levels of Proinflammatory Cytokines, Correlated With Blue and Red Signature Hub-Genes}

Because analysis of their transcriptome indicated that pSS pDCs are activated and/or primed for activation, we set out to confirm this on a functional level. As pro-inflammatory cytokine production is the hallmark of pDC activation, we measured the secretion of cytokines by unstimulated and TLRtriggered pDCs. Upon activation of pSS pDCs with loxoribine, we observed a remarkable mean increase in the production of IFN- $\alpha$ and IFN- $\beta$ compared to HC. Additionally, pSS pDCs produced more TNF- $\alpha$ in the unstimulated condition and more CXCL10 upon stimulation with CPG-C compared to HC pDCs. No differences were observed for IL-29/IFN$\lambda 1$ (Figure 5C) or IL-12 (very low, not shown). Interestingly, we observed significantly lower production of IFN- $\alpha$ and IFN- $\beta$ upon TLR7 triggering in pSS patients who were on immuno-suppressive treatment at the time of sample collection (Figure 5D, Supplementary Figure 5B).

We next investigated the association between hub-genes from the red and blue gene signatures with cytokine production. As treatment seemed to have an effect on cytokine production, treated patients were excluded from these analyses. Given the fact that the red signature contains the genes typically used to quantify the IFN-signature, we assessed differences in cytokine production between IFNsignature positive and negative patients, but no differences were observed (Supplementary Figure 5C). However, we 


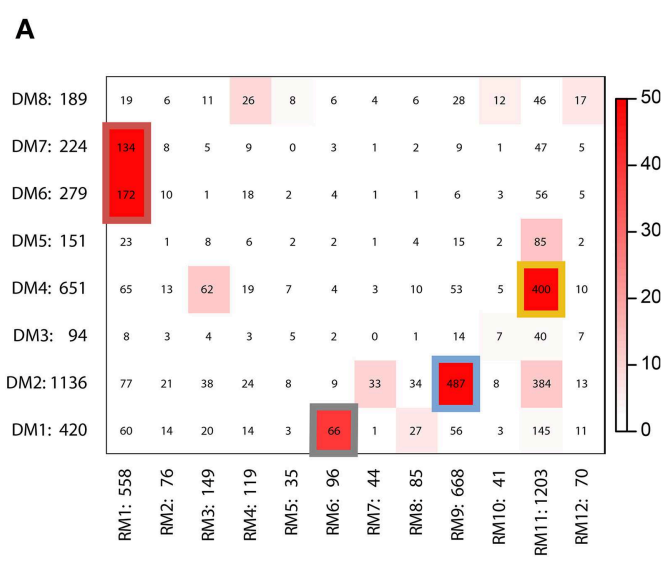

B

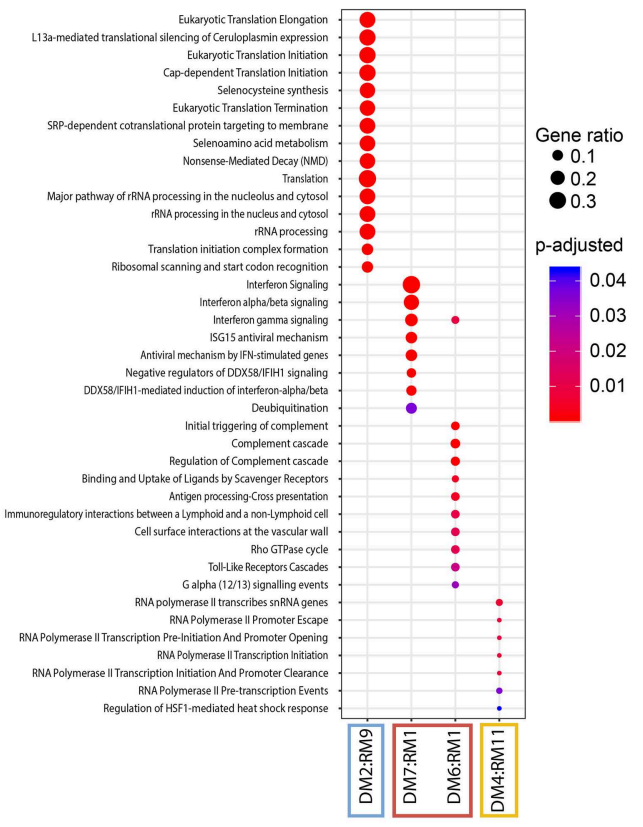

C

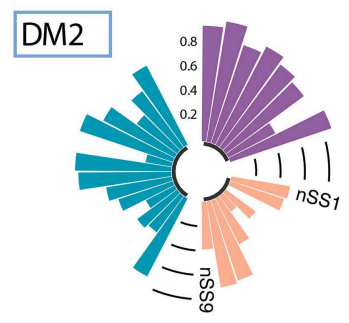

D
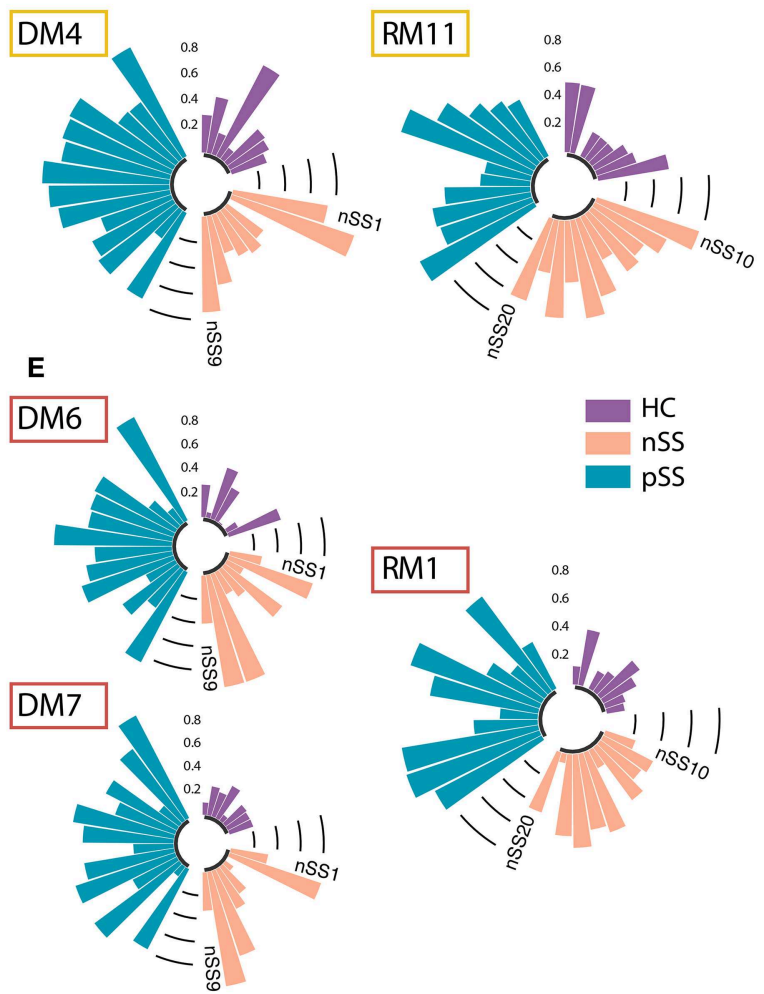

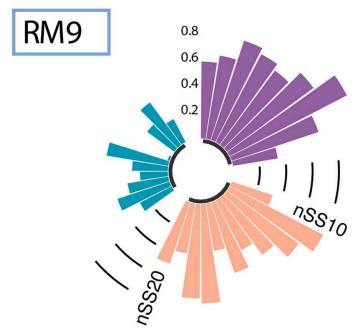

$\mathrm{HC}$
$\mathrm{nSS}$
$\mathrm{pSS}$

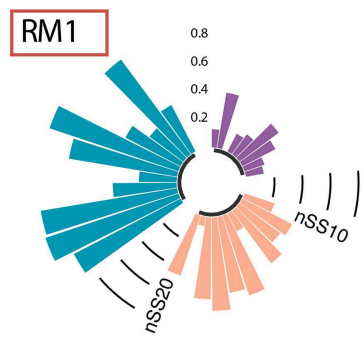

FIGURE 3 | Identification and functional annotation of replicated modules. WGCNA was performed to establish modules of co-expressed genes. To find the most robust and reproducible modules, the extent of overlap between the modules from the discovery and replication analysis was assessed. (A) depicts cross-tabulation of discovery modules (DM; rows) and replication modules (RM; columns). Each row and column is labeled by the corresponding module name and the number of replicated genes in the module. Within the table, numbers represent the count of genes present in the intersection of the corresponding row and column module. The table is color-coded by the Fisher exact test $p$-value. Five modules from the discovery cohort exhibited significant overlap with 4 modules from the replication cohort ( $p$ $<10^{-20}$ ). The genes in these replicated modules were designated as gene signatures gray (DM1:RM6), blue (DM2:RM9), yellow (DM4:RM11), and red (DM6+DM7:RM1). Go-term pathway enrichment was used for functional annotation of these signatures, there was no result for the gray signature. Dot-size depicts the fraction of the genes within the pathway that is enriched, color indicates $p$-value of the enrichment (B). Eigengenes (the first principal component) of the blue (C), yellow (D), and red (E) signatures are depicted, each bar represents an individual donor and values are in the range [0,1], where 0 represents the minimum and 1 represents the maximum value.

did observe significant correlations between the continuous IFN-score, reflecting upregulation of the genes in the red signature, and cytokine production for all four conditions where differences between pSS and HC donors were observed (IFN- $\alpha$ and IFN $-\beta$ upon stimulation with loxoribine; TNF- $\alpha$ without stimulation; CXCL10 with CPG-C). Similarly, the expression of the three blue signature hub-genes (RPL11, RPL27, RPS11) correlated with cytokine production (Figure 5E, Supplementary Figure 6). Finally, we observed associations between salivary gland inflammation and cytokine production
(Figure 5F), linking circulating pDC dysregulation to local immunopathology.

\section{Machine Learning Provides Proof of Concept for Harnessing pDC Transcriptional Data as Tool for Patient Stratification}

To assess whether transcriptional data from pDCs could be used for patient stratification, we developed a discriminative 

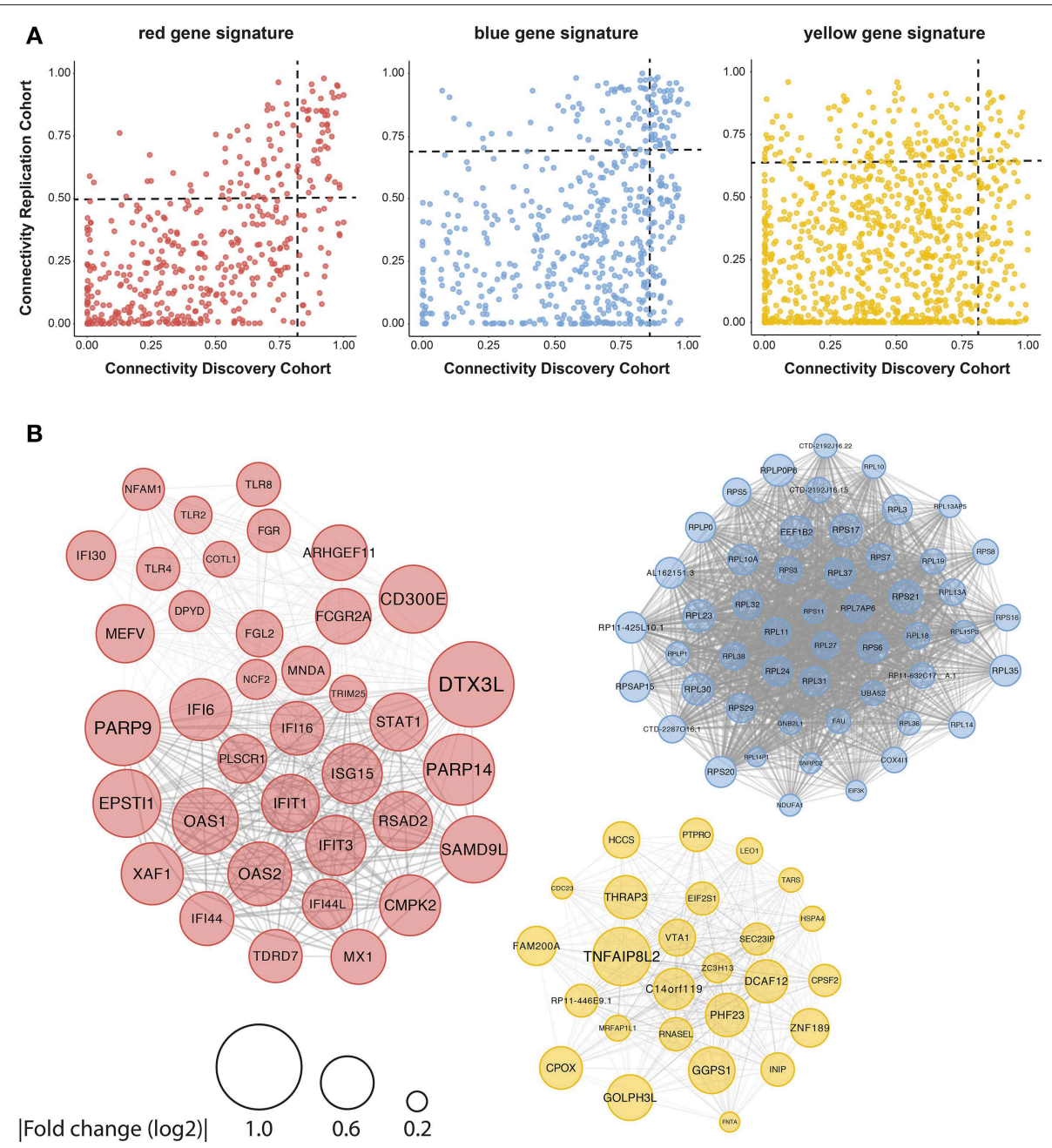

FIGURE 4 | Identification of consistently well-connected hub-genes in identified gene signatures. The connectivity of each gene within the red, blue, and yellow signatures is plotted for both the discovery and replication analysis. For each gene, the connectivity represents the sum of connection strengths with its neighbors in a given module. We normalized the connectivity for each module in the range [0, 1], where 0 represents the minimum and 1 represents the maximum connectivity in the module. Genes with a connectivity above the 75th percentile in both discovery and replication analysis were considered to be hub-genes, the 75th percentile cut-offs for both cohorts are shown with a black dotted line (A). (B) The hub genes were plotted based on the data from the replication cohort, the width of the edges between the nodes reflects the strength of their correlation and the size of each node shows the mean fold-change in expression between pSS and $\mathrm{HC}$ donors.

classifier using the robust and consistent transcriptional signature of pSS pDCs. Using machine learning, we selected 467 genes from the gene signatures that provided the most discriminative signal between pSS patients and HCs. (Figure 6A, Supplementary Figure 7). This selection allowed us to develop a support-vector machine model with 100\% sensitivity and $80 \%$ specificity in discriminating between pSS and HCs, underlining their transcriptional dissimilarity. Interestingly, when the nSS data were entered into the model, several donors were designated as "pSS-like" (prediction confidence $>75 \% ; n=7$ ).

Patient inclusion was based on the 2002 AECG classification criteria (21) in addition to clinical evaluation by an experienced rheumatologist. After sample collection for this study had finished, new Sjögren's syndrome classification-criteria were established with improved sensitivity and suitability for detection of patients at early stages of disease $(22,39)$. In this context, we wondered whether part of the nSS patients that our prediction model designated as pSS-like would meet these new classification-criteria at follow-up. For this, we invited all twenty included nSS donors for clinical re-evaluation, to which fourteen patients agreed. Five pSS-like donors and nine of the other nSS patients ("Reference" group; $<75 \%$ prediction confidence) were clinically re-evaluated at (median [IQR]) 3.5 [2.9-3.9] years after initial sample collection. At follow-up, three of the pSS-like patients (60\%) met the new pSS classification-criteria; this was true for only two of the donors from the reference group (22\%) (Figure 6B), suggesting that $\mathrm{pDC}$ transcriptional data may hold promise for patient stratification. 


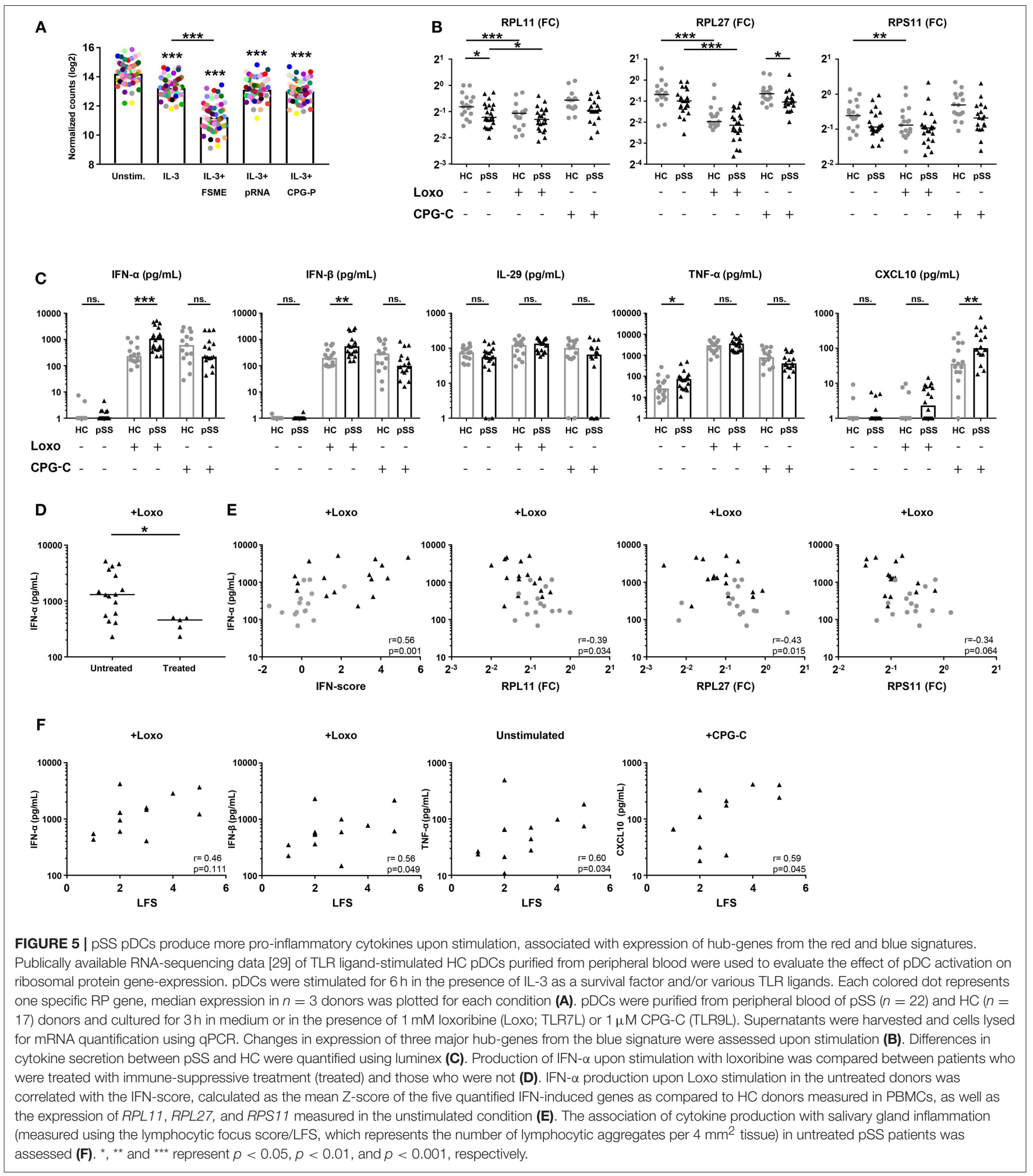

\section{DISCUSSION}

We here exploited RNA-sequencing to provide in-depth transcriptional analysis of pDCs from patients with pSS and nSS. The use of two independent cohorts of patients and controls allowed us to define robustly reproducible signatures of consistently altered and co-expressed genes. This included the differentially expressed genes directly related to the IFNsignature and those independent of it. Using this approach, we corroborate the majority of previously reported transcriptional 

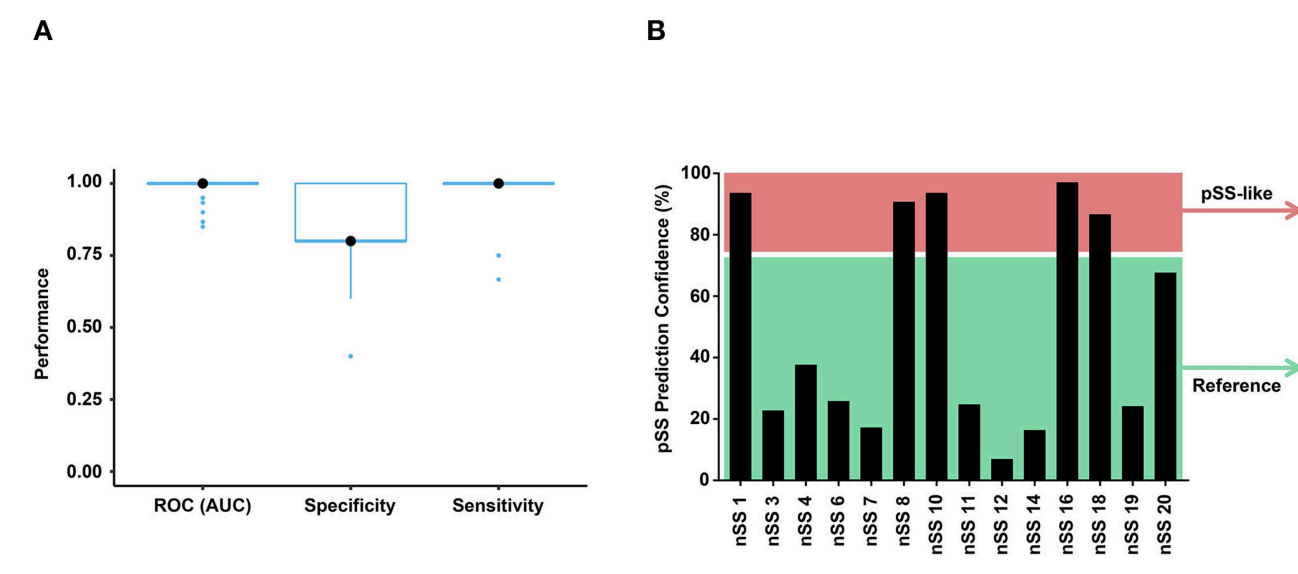

\begin{tabular}{|c|c|c|c|c|c|}
\hline Donor & $\begin{array}{c}\text { Meets } 2016 \\
\text { Criteria }\end{array}$ & $\underset{\left(/ 4 \mathrm{~mm}^{2}\right)}{\text { LFS }}$ & $\begin{array}{c}\text { Anti-Rol } \\
\text { SSA }\end{array}$ & $\begin{array}{l}\text { Schirmer } \\
\text { (15min) }\end{array}$ & $\begin{array}{l}\text { UWs } \\
\text { (15min) }\end{array}$ \\
\hline nSS1 & yes & 1,0 & neg & 4 & 0,0 \\
\hline nSS8 & no & 0,0 & neg & 18 & 4,5 \\
\hline nSS10 & yes & 0,6 & pos & 0 & 0,3 \\
\hline nSS16 & yes & 0,5 & pos & 3 & 0,1 \\
\hline nSS18 & no & 0,9 & neg & 12 & 0,1 \\
\hline Donor & $\begin{array}{c}\text { Meets } 2016 \\
\text { Criteria }\end{array}$ & $\underset{\left(/ 4 \mathrm{~mm}^{2}\right)}{\text { LFS }}$ & $\begin{array}{c}\text { Anti-Rol } \\
\text { SSA }\end{array}$ & $\begin{array}{l}\text { Schirmer } \\
\text { (/5min) }\end{array}$ & $\begin{array}{l}\text { UWs } \\
\text { (15min) }\end{array}$ \\
\hline nSS3 & no & 0,0 & neg & 3 & 0,6 \\
\hline nSS4 & no & 0,7 & neg & 40 & 0,6 \\
\hline nSS6 & no & 0,2 & neg & 4 & 1,0 \\
\hline nSS7 & yes & 1,3 & neg & 4 & 4,5 \\
\hline nSS 11 & no & 0,0 & pos & 24 & 1,7 \\
\hline nSS12 & yes & 1,1 & neg & 4 & 0,1 \\
\hline nSS14 & no & 0,6 & neg & 18 & 0,8 \\
\hline nSS 19 & no & 0,0 & pos & 8 & 1,6 \\
\hline$n S S 20$ & no & 0,0 & neg & 16 & 0,2 \\
\hline
\end{tabular}

FIGURE 6 | Prediction model discriminates between pSS and HC donors and identifies group of nSS patients more likely to meet the newest classification-criteria. After batch correction of the sequencing data from both cohorts, a selection of 467 genes from the replicated gene signatures was used to build a discriminative classifier that distinguishes between pSS and HC donors. (A) shows the performance of the discriminative classifier as validated using 10-fold cross validation. The transcriptional data of the nSS patients from both cohorts were entered into the classifier and a set of "pSS-like" donors (prediction confidence > 75\%) was identified. nSS patients with lower prediction confidence (<75\%) were included as "Reference" group. Fourteen nSS donors from either group were clinically re-evaluated in the context of the new 2016 classification-criteria for pSS at (median [IQR]) 3.5 [2.9-3.9] years after initial sample collection (B).

alterations in pDCs from a smaller cohort of IFN-signature positive pSS patients (19). Relevant exceptions include MYD88, TLR7, and TLR9; we did observe clear correlations of TLR7 and MYD88 with the IFN-score in both cohorts, supporting the published data (19), but we did not observe evidence for TLR9 downregulation in pSS patients or IFN-signature positive patients. Importantly, our data greatly expand upon the existing literature and identify a large set of novel genes associated with the IFN-signature in pSS pDCs. Some of these genes (e.g., DTX3L, PLSCR1, FCGR2A) are top hits in published studies in pSS (40-42). FCGR2A is of particular interest, as it is associated with internalization of immune complexes by pDCs $(43,44)$, which is a likely pathway by which pDCs are activated in pSS. Others are known IFN-induced genes that were not yet described in pSS (e.g., PARP14, TDRD7) with functions in antiviral immunity and regulation of type-I IFN production $(45,46)$. In addition, the red gene signature includes TLR2, TLR4, and $T L R 8$, which are not prominent $\mathrm{pDC}$ receptors (8) but may play a role in $\mathrm{pDC}$ activation under specific conditions, such as during viral infection or in inflamed tissues (47-49).

The yellow gene signature importantly contains CCR5, which is a chemokine receptor that is important for effective migration of pDCs to inflamed tissues and lymphnodes $(35,50,51)$. We confirmed the upregulation of CCR 5 in pSS pDCs on the protein level and in view of the increased levels of its ligands CCL3 and CCL4 in saliva of pSS patients (52), this receptor may mediate the migration of pDCs toward the salivary glands in pSS $(15,17,18)$. Furthermore, the yellow signature contains several hub genes involved in the regulation of apoptosis (e.g., DCAF12, TNFAIP8L2) as well as the transcription factors SP1 and TP53, which are central regulators of this process (53-55). The aberrant regulation of apoptosis may contribute to the decreased numbers of pDCs in the blood of patients with pSS (15). For the gray signature, functional annotation using pathway enrichment did not indicate any specific function. As pathway enrichment only considers known processes, novel data on these genes may shed more light on the function of the gray signature in the future.

The blue gene signature contains the majority of ribosomal protein genes as well as a range of ribosomal co-factors and is primarily involved in translation. To our knowledge, this is the first study that describes a broadly decreased expression of ribosomal proteins in pDCs in any setting. Using a published dataset (29) and in vitro pDC cultures, we show that this broad RP downregulation is associated with $\mathrm{pDC}$ activation via TLR7. In addition to translation, RPs have a broad range of cellular functions, including regulation of cytokine signaling and the preferential translation of specific (viral) transcripts (56-60). Furthermore, this signature is associated with nonsense-mediated decay, which is an important qualitycontrol mechanism that prevents disease by blocking translation of faulty transcripts and regulates important cellular processes by targeting intact mRNA (61). As such, this gene signature potentially has far-reaching consequences on pDC function. Interestingly, downregulation of RPs can be used by antigenpresenting cells to increase their relative abundance of exogenous antigen, and thus promote cross-presentation of viral peptides rather than endogenous peptides to CD8 T cells (38), which may be an ongoing process in pSS pDCs. The fact that TLR7L stimulation enables pDCs to effectively (cross-) present antigen $(62,63)$ corroborates the link between broad downregulation of RPs observed upon TLR ligation and cross-presentation of antigen. Future studies that combine transcriptomic data with proteomic data should shed light on the full extent of RP dysregulation in pSS pDCs. 
On the basis of [1] gene-set enrichment data indicating that patient pDCs are similar to TLR-triggered pDCs; [2] the downregulation of RP genes upon pDC activation, mimicking the blue signature; [3] evidence of signaling downstream of the type-I IFN receptor in the red signature, we conclude that pDCs from patients with pSS, and to a lesser extent nSS, have an activated phenotype at the gene expression level. As stimulation with even very low levels of type-I IFN results in enhanced cytokine production by pDCs upon TLR triggering and is required for optimal pDC activation (9-11), we hypothesized that patient pDCs are primed for enhanced cytokine production. To functionally validate this, we activated pDCs via TLR7 and TLR9 in a short culture of $3 \mathrm{~h}$ to minimize any effects of secreted type-I IFNs [produced after $\sim 2 \mathrm{~h}$ of culture (10)] on the transcriptome of the cultured cells. Indeed, pSS pDCs produced markedly higher type-I IFN levels, which correlated with the IFN-score and the expression of hub-genes from the blue signature, indicating that their transcriptome underpins the enhanced cytokine production. Interestingly, treatment was associated with reduced type-I IFN production by $\mathrm{pDCs}$ and seemed to affect the pDCs at the transcriptional level as well, as three of the four pSS patients misclassified in the prediction model were treated at time of sampling. Hydroxychloroquine was the most often used drug in these cohorts, and these findings are in-line with its effects on circulating immune cells as well as cultured cells described in literature $(19,64,65)$.

The activated phenotype and enhanced production of pro-inflammatory cytokines by $\mathrm{pSS}$ pDCs can substantially affect salivary gland inflammation. Type-I IFNs promote B cell-hyperactivity by driving B cell expansion, migration, and differentiation $(4,18,66,67)$. In addition, type-I IFNs stimulate lymphocyte attraction and expansion via activation of classical dendritic cells and other immune cells (68-70). The correlation between pDC cytokine production and the lymphocytic focus score corroborates the relevance of the altered pDC function for local inflammation. Considering the increased expression of CCR5 on pSS pDCs and the presence of its ligands CCL3 and CCL4 in saliva of pSS patients (52), this receptor may act as the link between circulating $\mathrm{pDCs}$ and the salivary glands in pSS $(15,17,18)$.

nSS patients form a poorly studied patient group with similar levels of dryness compared to pSS patients, but limited signs of (local) autoimmunity. To examine whether pDC dysregulation may play a role in these patients we included them in this study. Our data indicate that pDCs from nSS patients are similarly but less strongly activated compared to those from pSS patients on a group level. A set of nSS patients, who all did not meet the 2002 classification-criteria (21) at initial sample collection, was defined as pSS-like by our discriminative model and a relatively large fraction of them met the newest classificationcriteria (22) at follow-up. A limitation of this part of the study is that we were unable to include ocular staining in our clinical assessment. In addition, changes may have occurred in these patients in the time-period between initial sampling and followup. For instance, an increased focus score was observed in several donors compared to baseline. However, the relative effects of the enhanced sensitivity of the new criteria and any evolution of clinical manifestations are difficult to estimate. Nevertheless, our model discriminates between pSS and HC donors with high accuracy and additionally identifies a group of nSS patients of whom a relatively large fraction meets the more sensitive 2016 criteria at follow-up. As such, we provide proof of concept for further exploration of pDC transcriptional data as potential tool for patient stratification in sicca patients in future studies.

Concluding, we show that pSS pDCs are robustly distinct from $\mathrm{HC}$ pDCs, have an activated transcriptional phenotype, and are primed for increased pro-inflammatory cytokine production. nSS patients form an intermediate group in which some patients are molecularly similar to pSS patients in concordance with the most recent classification-criteria. In addition, the expression of hub genes from the two major identified gene signatures correlates with cytokine production, indicating that the transcriptional dysregulation of pSS pDCs underpins their enhanced cytokine production. These data substantiate the notion that $\mathrm{pDCs}$ play a role in pSS immunopathology and support studies that target pDCs, type-I IFNs, or IFN-signaling in pSS.

\section{DATA AVAILABILITY}

The RNA sequencing datasets generated for this study can be found in the GEO database at https://www.ncbi.nlm.nih.gov/geo/ - GSE135635. The raw data supporting the conclusions of this manuscript will be made available by the authors, without undue reservation, to any qualified researcher.

\section{ETHICS STATEMENT}

The medical ethics committee of the UMC Utrecht approved the study (METC; nr 13-697), all patients gave their written informed consent.

\section{AUTHOR CONTRIBUTIONS}

$\mathrm{MH}, \mathrm{AP}, \mathrm{SB}, \mathrm{MR}, \mathrm{AK}, \mathrm{JR}$, and TR were involved in conception and design of the study. $\mathrm{MH}, \mathrm{SB}, \mathrm{SH}, \mathrm{CB}, \mathrm{EH}$, and $\mathrm{AK}$ were involved in data acquisition. $\mathrm{MH}, \mathrm{AP}, \mathrm{SB}, \mathrm{NS}, \mathrm{AK}, \mathrm{JR}$, and TR were involved in data analysis and interpretation. $\mathrm{MH}$ and AP drafted the manuscript. All authors revised the manuscript critically for important intellectual content and approved the submitted version.

\section{FUNDING}

MH was supported by the Dutch Arthritis Association (ReumaNederland; Grant nr 17-2-301). AP was funded by the Netherlands Organization for Scientific Research (NWO; Grant nr 016.Veni.178.027). TR received research funding from GlaxoSmithKline. The funding sources had no role in study design; data collection, analysis, and interpretation; writing the report; or in the decision to submit the article for publication. 


\section{ACKNOWLEDGMENTS}

The authors would like to thank Kim van der WurffJacobs and Arno Concepcion for help with sample collection, Jennifer Sarikaya for assistance with flow-cytometry experiments, and the Multiplex core facility of the UMC Utrecht for performing the Luminex measurements. We are grateful to Dr. Francesca Barone, Ana Lopes, Dr. Lucas van den Hoogen,

\section{REFERENCES}

1. Campos J, Hillen MR, Barone F. Salivary gland pathology in Sjögren's syndrome. Rheum Dis Clin North Am. (2016) 42:473-83. doi: 10.1016/j.rdc.2016.03.006

2. Brito-Zerón P, Baldini C, Bootsma H, Bowman SJ, Jonsson R, Mariette $\mathrm{X}$, et al. Sjögren syndrome. Nat Rev Dis Primers. (2016) 2:16047. doi: $10.1038 /$ nrdp. 2016.47

3. Wildenberg ME, van Helden-Meeuwsen CG, van de Merwe JP, Drexhage HA, Versnel MA. Systemic increase in type I interferon activity in Sjögren's syndrome: a putative role for plasmacytoid dendritic cells. Eur J Immunol. (2008) 38:2024-33. doi: 10.1002/eji.200738008

4. Brkic Z, Maria NI, van Helden-Meeuwsen CG, van de Merwe JP, van Daele PL, Dalm VA, et al. Prevalence of interferon type I signature in CD14 monocytes of patients with Sjogren's syndrome and association with disease activity and BAFF gene expression. Ann Rheum Dis. (2013) 72:728-35. doi: 10.1136/annrheumdis-2012-201381

5. Szczerba BM, Rybakowska PD, Dey P, Payerhin KM, Peck AB, Bagavant $\mathrm{H}$, et al. Type I interferon receptor deficiency prevents murine Sjogren's syndrome. J Dent Res. (2013) 92:444-9. doi: 10.1177/0022034513483315

6. Thorlacius GE, Wahren-Herlenius M, Rönnblom L. An update on the role of type I interferons in systemic lupus erythematosus and Sjögren's syndrome. Curr Opin Rheumatol. (2018) 30:471-81. doi: 10.1097/BOR.0000000000000524

7. Muskardin TLW, Niewold TB. Type I interferon in rheumatic diseases. Nat Rev Rheumatol. (2018) 14:214-28. doi: 10.1038/nrrheum. 2018.31

8. Swiecki M, Colonna M. The multifaceted biology of plasmacytoid dendritic cells. Nat Rev Immunol. (2015) 15:471-85. doi: 10.1038/nri 3865

9. Liao AP, Salajegheh M, Morehouse C, Nazareno R, Jubin RG, Jallal B, et al. Human plasmacytoid dendritic cell accumulation amplifies their type 1 interferon production. Clin Immunol. (2010) 136:130-8. doi: 10.1016/j.clim.2010.02.014

10. Kim S, Kaiser V, Beier E, Bechheim M, Guenthner-Biller M, Ablasser A, et al. Self-priming determines high type I IFN production by plasmacytoid dendritic cells. Eur J Immunol. (2014) 44:807-18. doi: 10.1002/eji.2013 43806

11. Saitoh S-I, Abe F, Kanno A, Tanimura N, Mori Saitoh Y, Fukui R, et al. TLR7 mediated viral recognition results in focal type I interferon secretion by dendritic cells. Nat Commun. (2017) 8:1592. doi: 10.1038/s41467-01701687-x

12. Boulé MW, Broughton C, Mackay F, Akira S, Marshak-Rothstein A, Rifkin IR. Toll-like receptor 9-dependent and -independent dendritic cell activation by chromatin-immunoglobulin G complexes. J Exp Med. (2004) 199:1631-40. doi: $10.1084 /$ jem. 20031942

13. Båve U, Nordmark G, Lövgren T, Rönnelid J, Cajander S, Eloranta M-L, et al. Activation of the type I interferon system in primary Sjögren's syndrome: a possible etiopathogenic mechanism. Arthritis Rheumat. (2005) 52:1185-95. doi: 10.1002/art.20998

14. Ainola M, Porola P, Takakubo Y, Przybyla B, Kouri VP, Tolvanen TA, et al. Activation of plasmacytoid dendritic cells by apoptotic particlesmechanism for the loss of immunological tolerance in Sjögren's syndrome. Clin Exp Immunol. (2018) 191:301-10. doi: 10.1111/cei. 13077
Maarten van der Kroef, and Dr. Jonas Kuipers for their scientific input.

\section{SUPPLEMENTARY MATERIAL}

The Supplementary Material for this article can be found online at: https://www.frontiersin.org/articles/10.3389/fimmu. 2019.02096/full\#supplementary-material
15. Vogelsang P, Brun JG, Oijordsbakken G, Skarstein K, Jonsson R, Appel S. Levels of plasmacytoid dendritic cells and type-2 myeloid dendritic cells are reduced in peripheral blood of patients with primary Sjogren's syndrome. Ann Rheum Dis. (2010) 69:1235-8. doi: 10.1136/ard.2009.118158

16. Mingueneau M, Boudaoud S, Haskett S, Reynolds TL, Nocturne G, Norton E, et al. Cytometry by time-of-flight immunophenotyping identifies a blood Sjögren's signature correlating with disease activity and glandular inflammation. J Allergy Clin Immunol. (2016) 137:1809-21.e12. doi: 10.1016/j.jaci.2016.01.024

17. Gottenberg J-E, Cagnard N, Lucchesi C, Letourneur F, Mistou S, Lazure T, et al. Activation of IFN pathways and plasmacytoid dendritic cell recruitment in target organs of primary Sjögren's syndrome. Proc Natl Acad Sci USA. (2006) 103:2770-5. doi: 10.1073/pnas.0510837103

18. Zhao J, Kubo S, Nakayamada S, Shimajiri S, Zhang X, Yamaoka K, et al. Association of plasmacytoid dendritic cells with B cell infiltration in minor salivary glands in patients with Sjögren's syndrome. Mod Rheumatol. (2015) 26:716-24. doi: 10.3109/14397595.2015.1129694

19. Maria NI, Steenwijk EC, IJpma AS, van Helden-Meeuwsen CG, Vogelsang P, Beumer W, et al. Contrasting expression pattern of RNA-sensing receptors TLR7, RIG-I and MDA5 in interferon-positive and interferon-negative patients with primary Sjögren's syndrome. Ann Rheum Dis. (2016) 76:721-30. doi: 10.1136/annrheumdis-2016-209589

20. Barturen G, Beretta L, Cervera R, Van Vollenhoven R, Alarcón-Riquelme ME. Moving towards a molecular taxonomy of autoimmune rheumatic diseases. Nat Rev Rheumatol. (2018) 14:75-93. doi: 10.1038/nrrheum. 2017.220

21. Vitali C, Bombardieri S, Jonsson R, Moutsopoulos HM, Alexander EL, Carsons SE, et al. Classification criteria for Sjögren's syndrome: a revised version of the European criteria proposed by the AmericanEuropean Consensus Group. Ann Rheum Dis. (2002) 61:554-8. doi: 10.1136/ard.61.6.554

22. Shiboski CH, Shiboski SC, Seror R, Criswell LA, Labetoulle M, Lietman TM, et al. 2016 American College of Rheumatology/European League Against Rheumatism classification criteria for primary Sjögren's syndrome: a consensus and data-driven methodology involving three international patient cohorts. Ann Rheum Dis. (2017) 76:9-16. doi: 10.1136/annrheumdis-2016-210571

23. Yates A, Akanni W, Amode MR, Barrell D, Billis K, Carvalho-Silva D, et al. Ensembl 2016. Nucleic Acids Res. (2016) 44:D710-6. doi: 10.1093/nar/gkv1157

24. Dobin A, Davis CA, Schlesinger F, Drenkow J, Zaleski C, Jha S, et al. STAR: ultrafast universal RNA-seq aligner. Bioinformatics. (2013) 29:15-21. doi: 10.1093/bioinformatics/bts635

25. Anders S, Pyl PT, Huber W. HTSeq-a Python framework to work with high-throughput sequencing data. Bioinformatics. (2015) 31:166-9. doi: 10.1093/bioinformatics/btu638

26. Risso D, Ngai J, Speed TP, Dudoit S. Normalization of RNA-seq data using factor analysis of control genes or samples. Nat Biotechnol. (2014) 32:896-902. doi: 10.1038/nbt.2931

27. Love MI, Huber W, Anders S. Moderated estimation of fold change and dispersion for RNA-seq data with DESeq2. Genome Biol. (2014) 15:550. doi: 10.1186/s13059-014-0550-8

28. Subramanian A, Tamayo P, Mootha VK, Mukherjee S, Ebert BL, Gillette MA, et al. Gene set enrichment analysis: a knowledge-based approach for interpreting genome-wide expression profiles. Proc Natl Acad Sci USA. (2005) 102:15545-50. doi: 10.1073/pnas.0506580102 
29. Mathan TSM, Textor J, Sköld AE, Reinieren-Beeren I, van Oorschot T, Brüning $\mathrm{M}$, et al. Harnessing RNA sequencing for global, unbiased evaluation of two new adjuvants for dendritic-cell immunotherapy. Oncotarget. (2017) 8:19879-93. doi: 10.18632/oncotarget.15190

30. Sergushichev A. An Algorithm for Fast Preranked Gene Set Enrichment Analysis Using Cumulative Statistic Calculation. bioRxiv. doi: 10.1101/060012

31. Langfelder P, Horvath S. WGCNA: an R package for weighted correlation network analysis. BMC Bioinform. (2008) 9:559. doi: 10.1186/1471-2105-9-559

32. Langfelder P, Luo R, Oldham MC, Horvath S. Is my network module preserved and reproducible? PLoS Comput Biol. (2011) 7:e1001057. doi: 10.1371/journal.pcbi.1001057

33. $\mathrm{Yu} \mathrm{G}, \mathrm{He} \mathrm{Q}-\mathrm{Y}$. ReactomePA: an R/Bioconductor package for reactome pathway analysis and visualization. Mol Biosyst. (2016) 12:477-9. doi: $10.1039 / \mathrm{c} 5 \mathrm{mb} 00663 \mathrm{e}$

34. Shannon P, Markiel A, Ozier O, Baliga NS, Wang JT, Ramage D, et al. Cytoscape: a software environment for integrated models of biomolecular interaction networks. Genome Res. (2003) 13:2498-504. doi: 10.1101/gr.1239303

35. Brewitz A, Eickhoff S, Dähling S, Quast T, Bedoui S, Kroczek RA, et al. CD8 $(+)$ T cells orchestrate pDC-XCR1(+) dendritic cell spatial and functional cooperativity to optimize priming. Immunity. (2017) 46:205-19. doi: 10.1016/j.immuni.2017.01.003

36. Lelouard H, Schmidt EK, Camosseto V, Clavarino G, Ceppi M, Hsu H-T, et al. Regulation of translation is required for dendritic cell function and survival during activation. J Cell Biol. (2007) 179:1427-39. doi: 10.1083/jcb.2007 07166

37. Ceppi M, Clavarino G, Gatti E, Schmidt EK, de Gassart A, Blankenship $\mathrm{D}$, et al. Ribosomal protein mRNAs are translationally-regulated during human dendritic cells activation by LPS. Immunome Res. (2009) 5:5. doi: 10.1186/1745-7580-5-5

38. Argüello RJ, Reverendo M, Gatti E, Pierre P. Regulation of protein synthesis and autophagy in activated dendritic cells: implications for antigen processing and presentation. Immunol Rev. (2016) 272:28-38. doi: 10.1111/imr. 12427

39. Tsuboi H, Hagiwara S, Asashima H, Takahashi H, Hirota T, Noma H, et al. Comparison of performance of the 2016 ACR-EULAR classification criteria for primary Sjögren's syndrome with other sets of criteria in Japanese patients. Ann Rheum Dis. (2017) 76:1980-5. doi: 10.1136/annrheumdis-2016210758

40. Song GG, Kim J-H, Seo YH, Choi SJ, Ji JD, Lee YH. Metaanalysis of differentially expressed genes in primary Sjogren's syndrome by using microarray. Hum Immunol. (2014) 75:98-104. doi: 10.1016/j.humimm.2013.09.012

41. Khuder SA, Al-Hashimi I, Mutgi AB, Altorok N. Identification of potential genomic biomarkers for Sjögren's syndrome using data pooling of gene expression microarrays. Rheumatol Int. (2015) 35:829-36. doi: 10.1007/s00296-014-3152-6

42. Imgenberg-Kreuz J, Sandling JK, Almlöf JC, Nordlund J, Signér L, Norheim KB, et al. Genome-wide DNA methylation analysis in multiple tissues in primary Sjögren's syndrome reveals regulatory effects at interferon-induced genes. Ann Rheum Dis. (2016) 75:2029-36. doi: 10.1136/annrheumdis-2015-208659

43. Båve U, Magnusson M, Eloranta M-L, Perers A, Alm GV, Rönnblom L. Fc gamma RIIa is expressed on natural IFN-alpha-producing cells (plasmacytoid dendritic cells) and is required for the IFN-alpha production induced by apoptotic cells combined with lupus IgG. J Immunol. (2003) 171: 3296-302. doi: 10.4049/jimmunol.171.6.3296

44. Means TK, Latz E, Hayashi F, Murali MR, Golenbock DT, Luster AD. Human lupus autoantibody-DNA complexes activate DCs through cooperation of CD32 and TLR9. J Clin Invest. (2005) 115:407-17. doi: 10.1172/JCI 23025

45. Caprara G, Prosperini E, Piccolo V, Sigismondo G, Melacarne A, Cuomo A, et al. PARP14 Controls the nuclear accumulation of a subset of type I IFN-inducible proteins. I Immunol. (2018) 200:2439-54. doi: 10.4049/jimmunol.1701117

46. Subramanian G, Kuzmanovic T, Zhang Y, Peter CB, Veleeparambil M, Chakravarti $\mathrm{R}$, et al. A new mechanism of interferon's antiviral action: induction of autophagy, essential for paramyxovirus replication, is inhibited by the interferon stimulated gene, TDRD7. PLoS Pathog. (2018) 14:e1006877. doi: 10.1371/journal.ppat.1006877

47. Hernández JC, Arteaga J, Paul S, Kumar A, Latz E, Urcuqui-Inchima S. Upregulation of TLR2 and TLR4 in dendritic cells in response to HIV type 1 and coinfection with opportunistic pathogens. AIDS Res Hum Retroviruses. (2011) 27:1099-109. doi: 10.1089/aid.2010.0302

48. Dasgupta S, Erturk-Hasdemir D, Ochoa-Reparaz J, Reinecker H-C, Kasper DL. Plasmacytoid dendritic cells mediate anti-inflammatory responses to a gut commensal molecule via both innate and adaptive mechanisms. Cell Host Microbe. (2014) 15:413-23. doi: 10.1016/j.chom.2014.03.006

49. Ah Kioon MD, Tripodo C, Fernandez D, Kirou KA, Spiera RF, Crow MK, et al. Plasmacytoid dendritic cells promote systemic sclerosis with a key role for TLR8. Sci Transl Med. (2018) 10:eaam8458. doi: 10.1126/scitranslmed.aam8458

50. Diacovo TG, Blasius AL, Mak TW, Cella M, Colonna M. Adhesive mechanisms governing interferon-producing cell recruitment into lymph nodes. J Exp Med. (2005) 202:687-96. doi: 10.1084/jem.20051035

51. Sawai CM, Sisirak V, Ghosh HS, Hou EZ, Ceribelli M, Staudt LM, et al. Transcription factor Runx2 controls the development and migration of plasmacytoid dendritic cells. J Exp Med. (2013) 210:2151-9. doi: $10.1084 /$ jem.20130443

52. Lee YJ, Scofield RH, Hyon JY, Yun P-Y, Lee H-J, Lee EY, et al. Salivary chemokine levels in patients with primary Sjogren's syndrome. Rheumatology. (2010) 49:1747-52. doi: 10.1093/rheumatology/keq121

53. Hwangbo D-S, Biteau B, Rath S, Kim J, Jasper H. Control of apoptosis by Drosophila DCAF12. Dev Biol. (2016) 413:50-9. doi: 10.1016/j.ydbio.2016.03.003

54. Padmavathi G, Banik K, Monisha J, Bordoloi D, Shabnam B, Arfuso F, et al. Novel tumor necrosis factor- $\alpha$ induced protein eight (TNFAIP8/TIPE) family: functions and downstream targets involved in cancer progression. Cancer Lett. (2018) 432:260-271. doi: 10.1016/j.canlet.2018. 06.017

55. Li H, Zhang Y, Ströse A, Tedesco D, Gurova K, Selivanova G. Integrated high-throughput analysis identifies Sp1 as a crucial determinant of p53mediated apoptosis. Cell Death Differ. (2014) 21:1493-1502. doi: 10.1038/cdd. 2014.69

56. Shi Z, Fujii K, Kovary KM, Genuth NR, Röst HL, Teruel MN, Barna M. Heterogeneous ribosomes preferentially translate distinct subpools of mRNAs genome-wide. Mol Cell. (2017) 67:71-83.e7. doi: 10.1016/j.molcel.2017. 05.021

57. Huang J-Y, Su W-C, Jeng K-S, Chang T-H, Lai MMC. Attenuation of $40 \mathrm{~S}$ ribosomal subunit abundance differentially affects host and HCV translation and suppresses HCV replication. PLoS Pathog. (2012) 8:e1002766. doi: 10.1371/journal.ppat.1002766

58. Mazumder B, Sampath P, Seshadri V, Maitra RK, DiCorleto PE, Fox PL. Regulated release of L13a from the $60 \mathrm{~S}$ ribosomal subunit as a mechanism of transcript-specific translational control. Cell. (2003) 115:18798. doi: 10.1016/s0092-8674(03)00773-6

59. Mukhopadhyay R, Jia J, Arif A, Ray PS, Fox PL. The GAIT system: a gatekeeper of inflammatory gene expression. Trends Biochem Sci. (2009) 34:324-31. doi: 10.1016/j.tibs.2009.03.004

60. Wan F, Weaver A, Gao X, Bern M, Hardwidge PR, Lenardo MJ. IKK $\beta$ phosphorylation regulates RPS3 nuclear translocation and NF-кB function during infection with Escherichia coli strain O157:H7. Nat Immunol. (2011) 12:335-43. doi: 10.1038/ni.2007

61. Nasif S, Contu L, Mühlemann O. Beyond quality control: the role of nonsense-mediated mRNA decay (NMD) in regulating gene expression. Semin Cell Dev Biol. (2018) 75:78-87. doi: 10.1016/j.semcdb.2017. 08.053

62. Mouriès J, Moron G, Schlecht G, Escriou N, Dadaglio G, Leclerc C. Plasmacytoid dendritic cells efficiently cross-prime naive $T$ cells in vivo after TLR activation. Blood. (2008) 112:3713-22. doi: 10.1182/blood-2008-03-146290

63. Kool M, Geurtsvankessel C, Muskens F, Madeira FB, van Nimwegen M, Kuipers $\mathrm{H}$, et al. Facilitated antigen uptake and timed exposure to TLR ligands dictate the antigen-presenting potential of plasmacytoid DCs. J Leukoc Biol. (2011) 90:1177-90. doi: 10.1189/jlb.0610342 
64. Sacre K, Criswell LA, McCune JM. Hydroxychloroquine is associated with impaired interferon-alpha and tumor necrosis factor-alpha production by plasmacytoid dendritic cells in systemic lupus erythematosus. Arthritis Res Ther. (2012) 14:R155. doi: 10.1186/ar3895

65. Hjorton K, Hagberg N, Israelsson E, Jinton L, Berggren O, Sandling JK, et al. Cytokine production by activated plasmacytoid dendritic cells and natural killer cells is suppressed by an IRAK4 inhibitor. Arthritis Res Ther. (2018) 20:238. doi: 10.1186/s13075-018-1702-0

66. Menon M, Blair PA, Isenberg DA, Mauri C. A regulatory feedback between plasmacytoid dendritic cells and regulatory b cells is aberrant in systemic lupus Erythematosus. Immunity. (2016) 44:683-97. doi: 10.1016/j.immuni.2016.02.012

67. Berggren O, Hagberg N, Alexsson A, Weber G, Rönnblom L, Eloranta M-L. Plasmacytoid dendritic cells and RNA-containing immune complexes drive expansion of peripheral B cell subsets with an SLE-like phenotype. PLoS ONE. (2017) 12:e0183946. doi: 10.1371/journal.pone. 0183946

68. Ito $\mathrm{T}$, Amakawa $\mathrm{R}$, Inaba $\mathrm{M}$, Ikehara $\mathrm{S}$, Inaba $\mathrm{K}$, Fukuhara $\mathrm{S}$. Differential regulation of human blood dendritic cell subsets by IFNs. J Immunol. (2001) 166:2961-2969. doi: 10.4049/jimmunol.166. 5.2961

69. Lapenta C, Santini SM, Spada M, Donati S, Urbani F, Accapezzato D, et al. IFN-alpha-conditioned dendritic cells are highly efficient in inducing cross-priming CD8(+) T cells against exogenous viral antigens. Eur J Immunol. (2006) 36:2046-2060. doi: 10.1002/eji.200535579

70. Sozzani S, Del Prete A, Bosisio D. Dendritic cell recruitment and activation in autoimmunity. J. Autoimmunity. (2017) 85:126-40. doi: 10.1016/j.jaut.2017.07.012

Conflict of Interest Statement: TR is a principal investigator in the immune catalyst program of GlaxoSmithKline, which is an independent research program. He did not receive any financial support other than the research funding for the current project.

The remaining authors declare that the research was conducted in the absence of any commercial or financial relationships that could be construed as a potential conflict of interest.

Copyright (C) 2019 Hillen, Pandit, Blokland, Hartgring, Bekker, van der Heijden, Servaas, Rossato, Kruize, van Roon and Radstake. This is an open-access article distributed under the terms of the Creative Commons Attribution License (CC BY). The use, distribution or reproduction in other forums is permitted, provided the original author(s) and the copyright owner(s) are credited and that the original publication in this journal is cited, in accordance with accepted academic practice. No use, distribution or reproduction is permitted which does not comply with these terms. 